\title{
Knockdown of the mitochondria-localized protein p13 protects against experimental parkinsonism
}

\author{
Naoki Inoue $1,2,3$, Sae Ogura ${ }^{1}$, Atsushi Kasai ${ }^{1}$, Takanobu Nakazawa ${ }^{1,4}$, Kazuya Ikeda ${ }^{1}$, Shintaro Higashi ${ }^{1}$, \\ Ayako Isotani ${ }^{5,6}$, Kousuke Baba ${ }^{7}$, Hideki Mochizuki ${ }^{7}$, Harutoshi Fujimura ${ }^{8}$, Yukio Ago ${ }^{1}$, \\ Atsuko Hayata-Takano ${ }^{1,9}$, Kaoru Seiriki ${ }^{1,2}$, Yusuke Shintani ${ }^{1}$, Norihito Shintani ${ }^{1,{ }^{*}}$ (D) \& \\ Hitoshi Hashimoto ${ }^{1,9,10,11,{ }^{* *} \text { (iD }}$
}

\begin{abstract}
Mitochondrial dysfunction in the nigrostriatal dopaminergic system is a critical hallmark of Parkinson's disease (PD). Mitochondrial toxins produce cellular and behavioural dysfunctions resembling those in patients with PD. Causative gene products for familial PD play important roles in mitochondrial function. Therefore, targeting proteins that regulate mitochondrial integrity could provide convincing strategies for PD therapeutics. We have recently identified a novel $13-\mathrm{kDa}$ protein ( $p 13$ ) that may be involved in mitochondrial oxidative phosphorylation. In the current study, we examine the mitochondrial function of p13 and its involvement in PD pathogenesis using mitochondrial toxininduced PD models. We show that p13 overexpression induces mitochondrial dysfunction and apoptosis. p13 knockdown attenuates toxin-induced mitochondrial dysfunction and apoptosis in dopaminergic SH-SY5Y cells via the regulation of complex I. Importantly, we generate p13-deficient mice using the CRISPR/ Cas9 system and observe that heterozygous $p 13$ knockout prevents toxin-induced motor deficits and the loss of dopaminergic neurons in the substantia nigra. Taken together, our results suggest that manipulating p13 expression may be a promising avenue for therapeutic intervention in PD.
\end{abstract}

Keywords cell death; complex I; mitochondria; p13; Parkinson's disease Subject Categories Molecular Biology of Disease; Neuroscience DOI 10.15252/embr.201744860 | Received 19 July 2017| Revised 15 December 2017 | Accepted 21 December 2017| Published online 25 January 2018 EMBO Reports (2018) 19: e44860
See also: JS Valadas \& P Verstreken (March 2018)

\section{Introduction}

Parkinson's disease (PD), one of the most common motor disorders, is caused by the progressive and specific loss of nigrostriatal dopaminergic neurons [1,2]. Dopamine modulators are first-line therapeutics for $\mathrm{PD}$, but they face tolerability issues and have limited efficacy [3]. Therefore, there is a compelling need for novel therapeutic strategies for PD [4-6].

Although the aetiology of PD remains unclear, the involvement of mitochondrial dysfunction in PD has been increasingly convincing [7-10]. Mitochondrial complex I deficiencies are detected in postmortem nigrostriatal tissues of patients with PD [11-13]. Inhibitors of mitochondrial complex I such as rotenone and 1-methyl-4-tetramethyl-p-phenylenediamine (MPTP) induce several hallmarks of PD, including motor deficits and nigrostriatal dopaminergic cell death in rodents and primates [14-16]. Furthermore, mutations in mitochondrial DNA (mtDNA) leading to mitochondrial dysfunction have been considered a risk factor for PD $[17,18]$. The current model of PD proposes that defects in PTEN-induced putative kinase 1 (PINK1) and Parkin, well-known causative gene products for familial PD, cause an accumulation of dysfunctional mitochondria $[19,20]$. These findings argue that maintaining mitochondrial integrity could be a powerful and convincing strategy for PD treatment.

We have recently identified a novel $13-\mathrm{kDa}$ protein named p13, whose expression is decreased in pancreatic islets exposed to oxidative

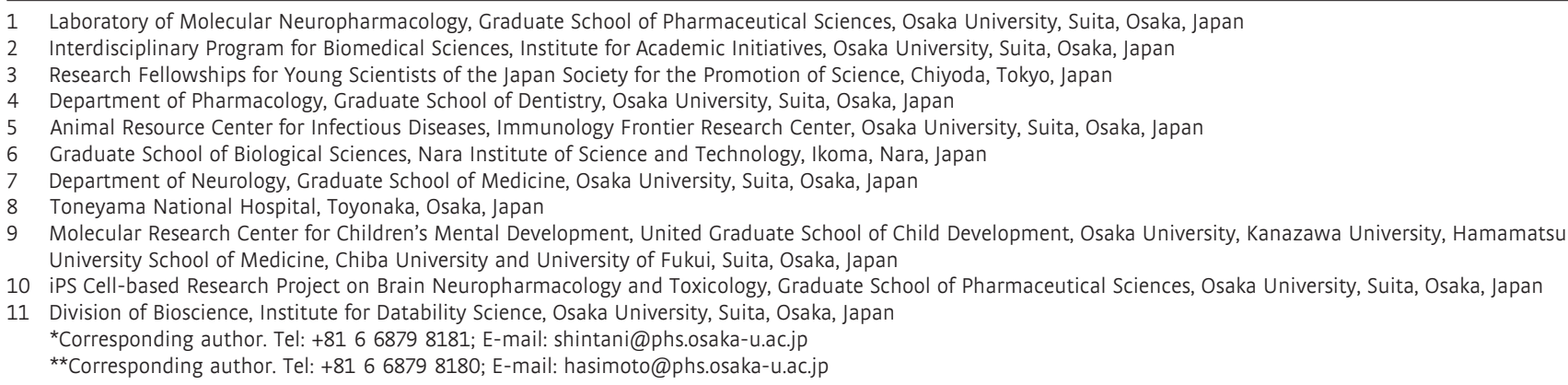


stress by a high-fat diet [21]. Oxidative stress is caused by the excessive accumulation of reactive oxygen species generated in dysfunctional mitochondria [22,23]. Recent proteomic analysis has identified human p13 (C7orf55) as a novel protein that may interact with mitochondrial proteins such as NDUFAB1, a complex I subunit, and ATPAF2, a complex V subunit [24]. Furthermore, p13 has been demonstrated to partially co-localize with mtDNA by immunostaining [25]. We therefore hypothesized that p13 is a regulator of mitochondrial function. However, the mitochondrial function of p13 and its involvement in the molecular pathogenesis of PD are currently unknown.

In the present study, we examined the roles of p13 in mitochondrial function in both in vitro and in vivo PD models. Our results suggest that the reduction in p13 expression acts as a protective factor against $\mathrm{PD}$ pathogenesis via the maintenance of mitochondrial function.

\section{Results and Discussion}

\section{p13 overexpression exacerbates rotenone-induced mitochondrial dysfunction and apoptosis in SH-SY5Y cells}

We found that p13 was co-localized with Hsp60, a mitochondrial matrix-localized protein in SH-SY5Y cells, a human neuroblastoma cell line (Fig 1A). Next, we measured mitochondrial membrane potential $(\Delta \Psi \mathrm{m})$ using tetramethylrhodamine ethyl ester perchlorate (TMRE), which is sensitive to $\Delta \Psi \mathrm{m}$. We found that p13 overexpression significantly decreased $\Delta \Psi \mathrm{m}$ compared with the levels measured in mock-infected cells (Fig 1B). The $\Delta \Psi \mathrm{m}$ decrease induced by rotenone, a mitochondrial complex I inhibitor, was exacerbated in p13overexpressed SH-SY5Y cells (Fig 1B). The signal of MitoTracker Green FM, which localizes to mitochondria regardless of $\Delta \Psi \mathrm{m}$, did not differ between mock- and p13-overexpressed cells under basal or rotenone-treated conditions (Fig 1C), suggesting that p13 overexpression does not affect mitochondrial mass. Because mitochondria play a key role in apoptosis $[26,27]$, we evaluated the effects of p13 overexpression on apoptosis induction by measuring the levels of cleavage of poly (ADP-ribose) polymerase (PARP). We observed that p13 overexpression significantly increased the levels of PARP cleavage in both the vehicle- and the rotenone-treated cells (Fig 1D). We also applied the terminal deoxynucleotidyl transferase (TdT)-mediated deoxyuridine triphosphate (dUTP) nick-end labelling (TUNEL) method to detect apoptotic cells. We found that the overexpression of p13 increased the number of TUNEL-positive cells under basal conditions and exacerbated the rotenone-induced increase in TUNEL-positive cells (Fig 1E). These data demonstrate that p13 overexpression induces mitochondrial dysfunction and apoptosis in SH-SY5Y cells.

\section{p13 knockdown prevents parkinsonian toxicant-induced mitochondrial dysfunction and apoptosis in SH-SY5Y cells}

We first performed subcellular fractionation experiments and observed that endogenous p13 was most abundant in the mitochondria-enriched fraction (Figs 2A and EV4B). Furthermore, to characterize the intramitochondrial localization of endogenous p13, we used digitonin fractionation, in which mitochondria were treated with various concentrations of digitonin for progressive membrane solubilization. We found that p13 showed a similar resistance to digitonin compared with the mitochondrial matrix marker Hsp60 (Fig 2B). Tom20 and Tim23, which are mitochondrial outer and inner membrane markers, respectively, are more sensitive to higher concentrations of digitonin than p13 or Hsp60 is (Fig 2B). These results suggest that p13 is mainly localized in the mitochondrial matrix. Based on the results from the overexpression experiments (Fig 1), we hypothesized that the downregulation of p13 expression prevents stress-induced mitochondrial dysfunction and apoptosis. We confirmed shRNA-mediated p13 knockdown in SH-SY5Y cells (Fig EV1A and B). We then evaluated the effects of p13 knockdown on rotenone-induced mitochondrial dysfunction and apoptosis in SH-SY5Y cells. In contrast to control knockdown, p13 knockdown significantly prevented the rotenone-induced decrease in $\Delta \Psi \mathrm{m}$ and increases in cleaved PARP and TUNEL-positive cells (Fig 2C-E). In the basal condition, the p13 knockdown did not affect the fluorescence intensity of TMRE, the cleavage of PARP or the percentage of TUNEL-positive cells (Fig 2C-E). Similar results were obtained using another shRNA construct (p13 shRNA \#2) targeting a different region of p13 (Fig EV1C-E). Furthermore, p13 knockdown attenuated the decrease in $\Delta \Psi \mathrm{m}$ and the release of lactate dehydrogenase (LDH), a marker of cell death, by the mitochondrial complex I inhibitor 1-methyl-4-phenylpyridine $\left(\mathrm{MPP}^{+}\right)$, a metabolite of MPTP (Fig EV1G and H), confirming the protective effects of p13 knockdown against complex-I-inhibitor-induced toxicity. In addition to the toxin-induced PD model, we also examined the effects of p13 knockdown using a non-toxic PD model in which PINK1 was knocked down (Fig EV1I) and found that p13 knockdown significantly prevented the decrease in $\Delta \Psi \mathrm{m}$ and the PARP cleavage in the PINK1-knockdown cells (Fig EV1J and K). Importantly, in rescue experiments (Fig $2 \mathrm{~F}$ and $\mathrm{G}$ ), the restoration of p13 expression in p13-knockdown cells significantly reversed the protective effects of p13 knockdown on PARP cleavage by rotenone (Fig $2 \mathrm{G}$ ), but the effect of the restoration of p13 expression on the rotenoneinduced decrease in $\Delta \Psi \mathrm{m}$ was modest (Fig $2 \mathrm{~F}$ ). These data suggest that p13 knockdown significantly prevents parkinsonian toxicantinduced mitochondrial dysfunction and apoptosis in SH-SY5Y cells.

Figure 1. p13 overexpression exacerbates rotenone-induced mitochondrial dysfunction and apoptosis in SH-SY5Y cells.

A Co-localization of overexpressed p13 and Hsp60, a mitochondrial matrix protein, in p13-infected cells. Nucleus was stained with Hoechst (blue). Scale bars, $10 \mu \mathrm{m}$.

$B, C$ Exacerbated rotenone-induced decrease in $\Delta \Psi \mathrm{m}$ but no change in mitochondrial mass in p13-infected cells. $\Delta \Psi \mathrm{m}$ and mitochondrial mass were determined by measuring the fluorescence levels of TMRE (B) and MitoTracker Green FM (C), respectively.

$D, E$ Exacerbated rotenone-induced apoptosis in p13-infected cells. Apoptosis levels were evaluated by measuring the increases in PARP cleavage (D) and in percentage of TUNEL-positive cells (E). The levels of cleaved PARP were normalized to those of $\beta$-actin (D). The percentage of TUNEL-positive cells was determined by TUNEL (green) and Hoechst (blue, a nuclear marker) staining (E). Representative images (left) and their quantification (right) were shown. Scale bar, $50 \mu \mathrm{m}$.

Data information: In all experiments, cells were infected with lentiviral vectors expressing mock or FLAG-tagged p13 (p13 o/e). Seventy-two hours after infection, cells were exposed to vehicle or $100 \mathrm{nM}$ rotenone for $24 \mathrm{~h}(\mathrm{~B}-\mathrm{E})$. p13 was detected using an antibody against p13. All data are presented as the mean \pm SEM ( $n=3$ ). ${ }^{\star} P<0.05,{ }^{* *} P<0.01$ by the Tukey-Kramer test. 
A

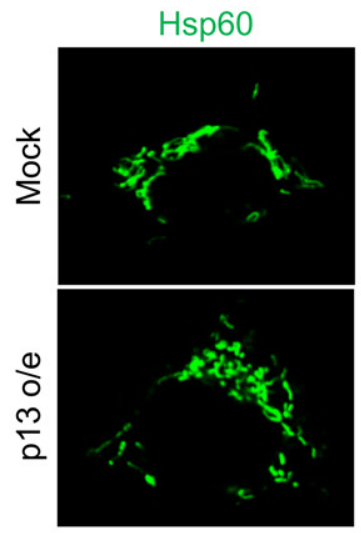

B
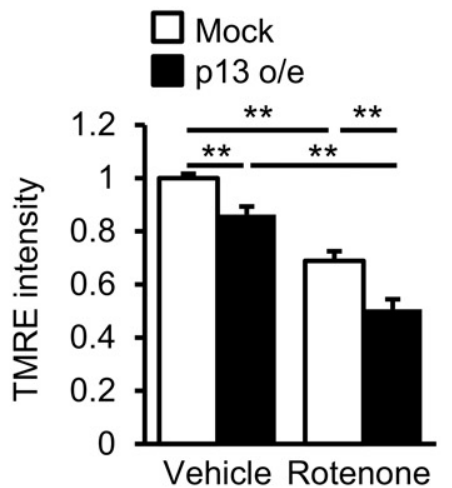

D

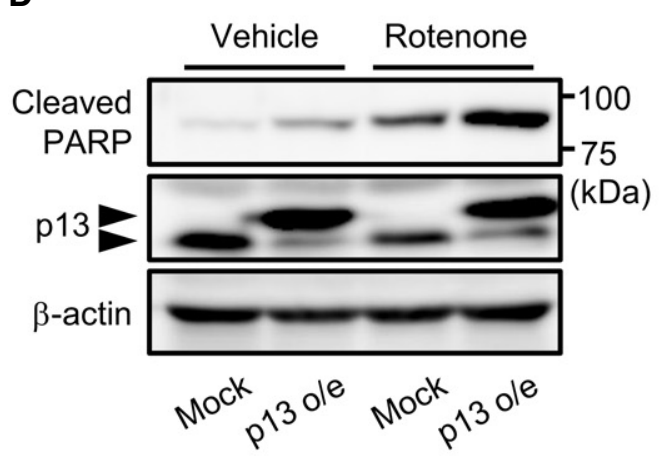

E

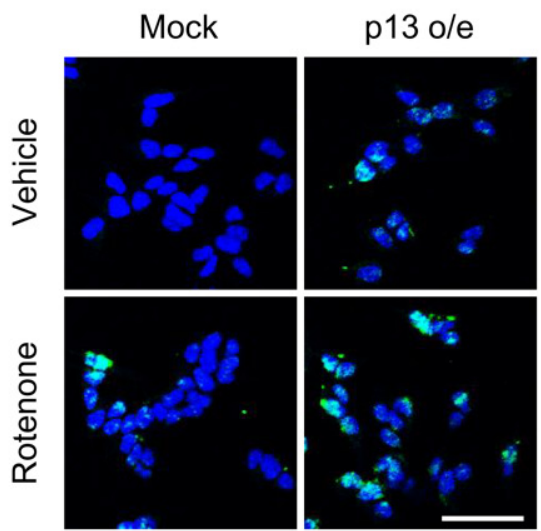

p13

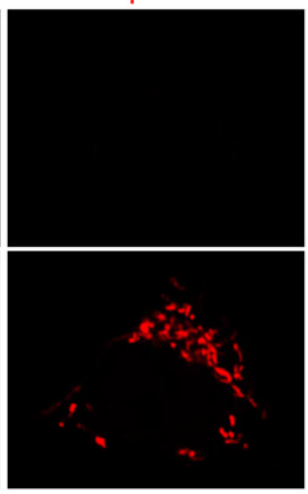

C $\square$ Mock

p13 o/e

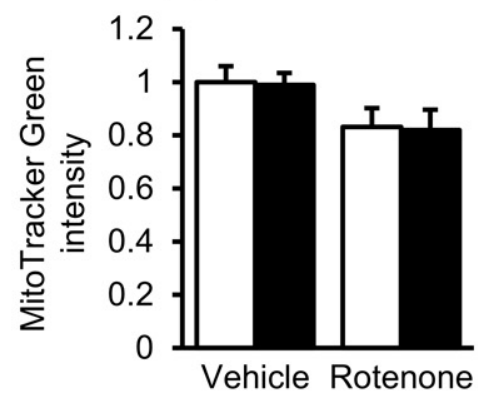

$\square$ Mock

p13 o/e
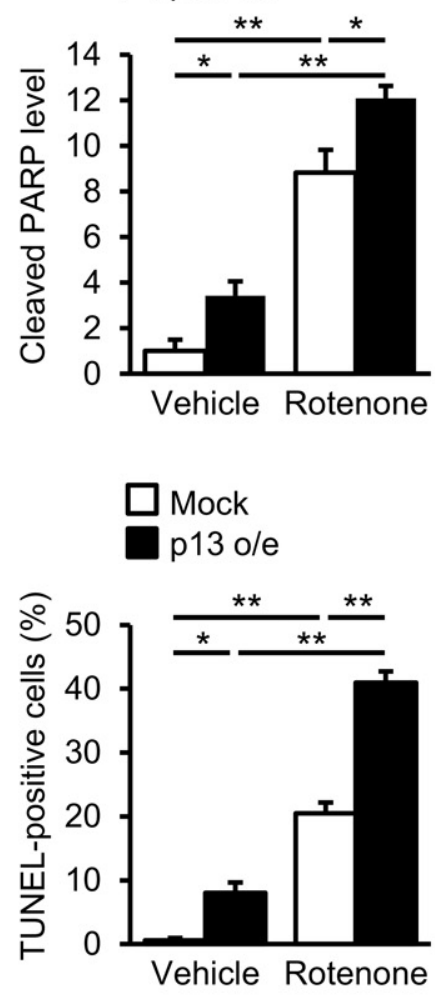

Figure 1. 
A

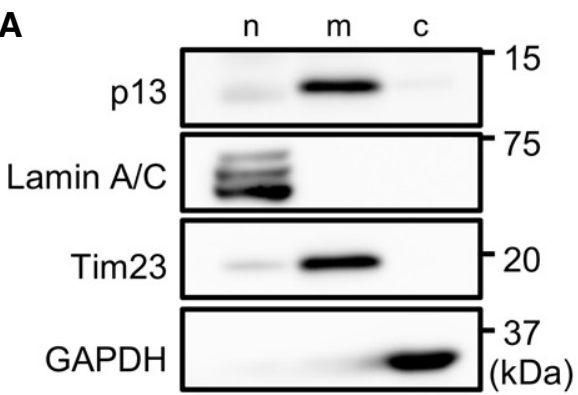

B

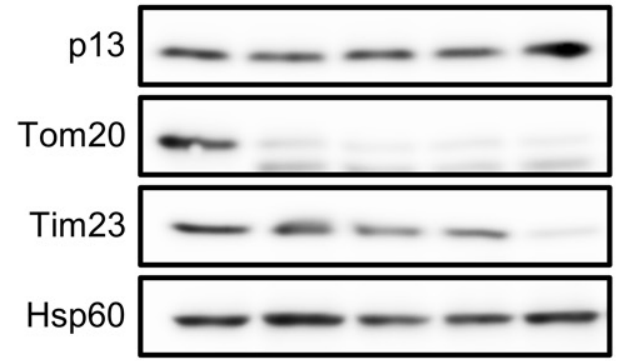

$\begin{array}{llllll}\text { Digitonin (\%) } & 0 & 0 & 0.01 & 0.05 & 0.1\end{array}$

ProK -++++

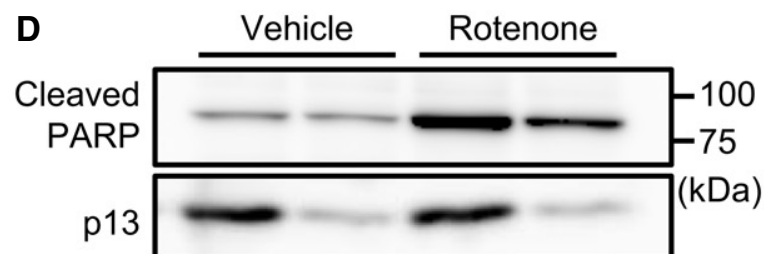

$\beta$-actin
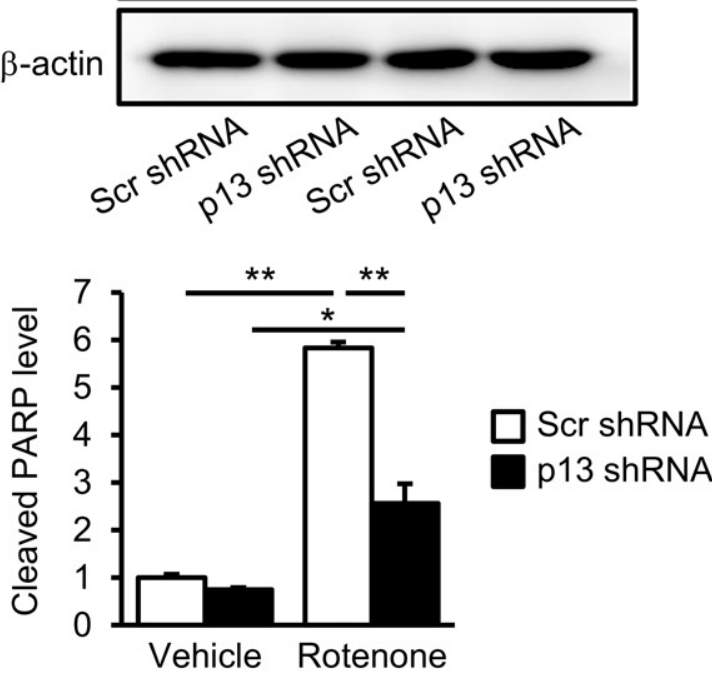

C

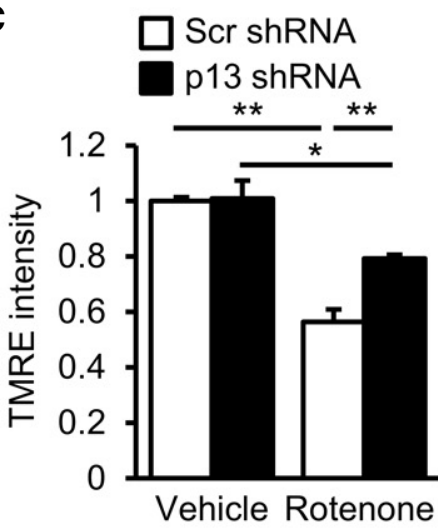

$\mathbf{F}$
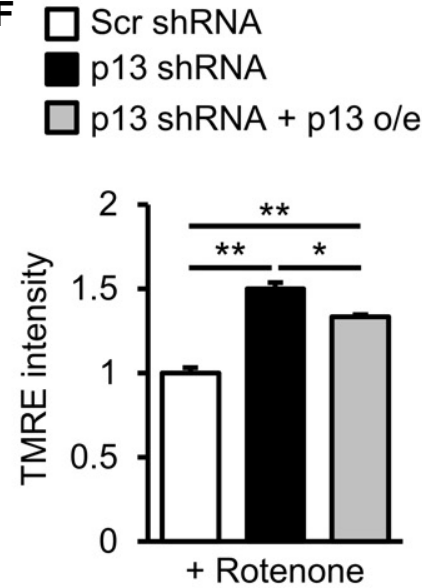

E Scr shRNA
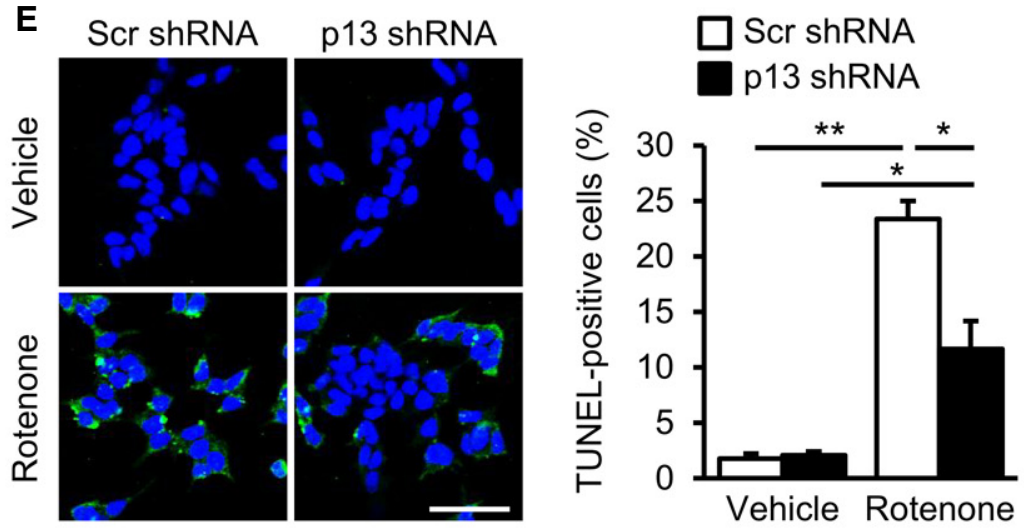

G

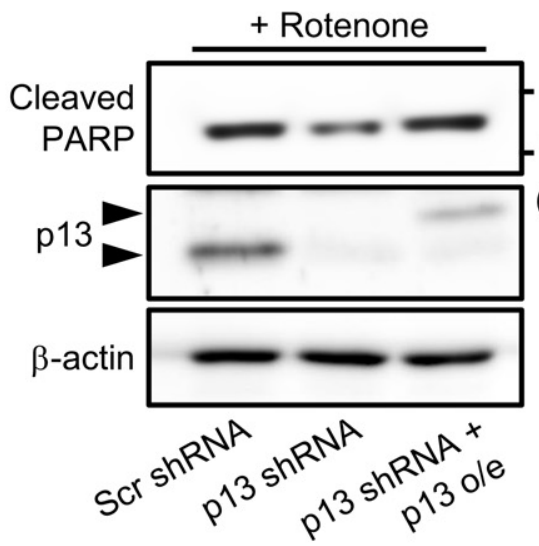

$\square$ Scr shRNA

p13 shRNA

100

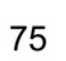

$\square \mathrm{p} 13$ shRNA + p13 o/e

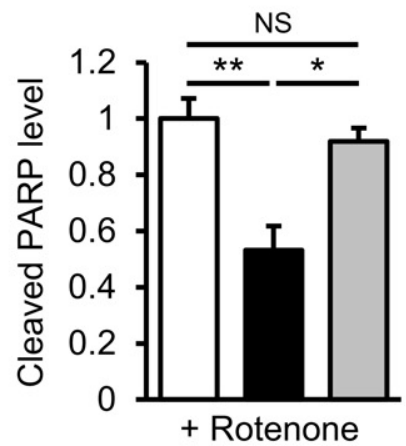

Figure 2. 
Figure 2. p13 knockdown prevents rotenone-induced mitochondrial dysfunction and apoptosis in SH-SY5Y cells.

A Distribution of p13 in the nuclear (n)-, mitochondria (m)- and cytosol (c)-enriched fractions of SH-SY5Y cells. The blot was probed with antibodies against p13, Lamin A/C (a nuclear marker), Tim23 (a mitochondrial marker) and GAPDH (a cytoplasmic marker).

B Mitochondrial localization of p13 in SH-SY5Y cells. Isolated mitochondria were treated with $20 \mu \mathrm{g} / \mathrm{ml}$ proteinase $\mathrm{K}$ (Pro $\mathrm{K}$ ) in the absence or presence of increasing concentrations of digitonin $(0.01,0.05$ and $0.1 \%$ ). Samples were subjected to Western blotting with antibodies against p13, Tom20 (outer mitochondrial membrane marker), Tim23 (inner mitochondrial membrane marker) and Hsp60 (mitochondrial matrix marker).

C-E Prevention of the rotenone-induced decrease in $\triangle \Psi m$ (C), increase in PARP cleavage (D) and increase in percentage of TUNEL-positive cells (E) in p13 shRNAinfected cells. Scale bar, $50 \mu \mathrm{m}$.

F, G Attenuation of the rotenone-induced decrease in $\triangle \Psi \mathrm{m}$ (F) and increase in PARP cleavage (C) by the restoration of p13 expression in p13 shRNA-infected cells. Arrowheads showed FLAG-tagged p13 (p13 o/e) and endogenous p13.

Data information: Seventy-two hours after infection with lentiviral vectors expressing scrambled shRNA (Scr shRNA) or p13 shRNA, cells were exposed to vehicle or 100 nM rotenone for $24 \mathrm{~h}(\mathrm{C}-\mathrm{C}) . \Delta \Psi \mathrm{m}$ was determined by measuring the TMRE fluorescence levels (C, F). The levels of cleaved PARP were normalized to those of $\beta$-actin (D, C). The percentage of TUNEL-positive cells was determined by TUNEL (green) and Hoechst (blue, a nuclear marker) staining (E). Representative images (upper, D; left, E, C) and their quantification (lower, D; right, E, G) were shown. All data are presented as the mean $\pm \operatorname{SEM}(n=3)$. ${ }^{*} P<0.05$, ${ }^{* \star} P<0.01$ by the Tukey-Kramer test. NS, not significant.

Currently, it is unclear why, in contrast to p13 overexpression, p13 knockdown did not affect the fluorescence intensity of TMRE and the cleavage of PARP in the basal condition. We assume, however, several possibilities to reconcile the discrepancy. (i) A small amount of residual p13 protein after p13 knockdown may keep mitochondrial function in the basal condition. (ii) Other mitochondrial factors may compensate for the effect of p13 knockdown in the basal condition.

\section{p13 knockdown maintains complex I activity independently of PINK1-associated autophagy in rotenone-treated SH-SY5Y cells}

Recent reports have shown that p13 may interact with mitochondrial complex I and mtDNA [24,25]; however, it is unclear whether p13 is involved in their functions. We first examined the effects of p13 knockdown on complex I activity in the rotenone-treated SHSY5Y cells. Interestingly, p13 knockdown significantly prevented the rotenone-induced decrease in complex I activity compared with that observed in scrambled shRNA-infected cells (Figs 3A and EV1F). We next examined the co-immunoprecipitation study and found that the overexpressed p13 co-precipitated well with complex $\mathrm{I}$, as revealed by an immunoblot for nicotinamide adenine dinucleotide dehydrogenase (ubiquinone) $1 \beta$ subcomplex 8 (NDUFB8), a mitochondrial complex I subunit, in SH-SY5Y cells (Fig 3B). Endogenous p13 also co-precipitated with complex I proteins in $\mathrm{SH}$ SY5Y cells (Fig 3C). Immunocytochemistry showed that the overexpressed p13 was co-localized with mitochondrial complex I (Fig EV2A). We then examined the effects of p13 knockdown on complex I assembly in SH-SY5Y cells and found that p13 knockdown prevented the rotenone-induced impairment of complex I assembly (Fig 3D). We then examined the expression levels of mitochondrial mRNAs encoded by mtDNA in p13-knockdown rotenone-treated SH-SY5Y cells. There was no significant difference in mitochondrial mRNA expression levels between scrambled shRNAand p13 shRNA-infected rotenone-treated SH-SY5Y cells (Fig EV2B). These results suggest that the interaction between p13 and complex I but not mtDNA is necessary to prevent mitochondrial dysfunction.

A popular topic in PD pathogenesis research is PINK1/Parkinmediated autophagy [28]. PINK1 accumulates on the mitochondrial outer membrane following the loss of $\Delta \Psi \mathrm{m}[29,30]$ and, in conjunction with Parkin, helps to eliminate damaged mitochondria by autophagy [31-33]. To examine whether the autophagy machinery is involved in the protective effects of p13 knockdown, we evaluated the mitochondrial accumulation of PINK1 and autophagy induction in rotenone-treated SH-SY5Y cells. We observed that PINK1 accumulation was not stimulated by rotenone regardless of the manipulation of p13 expression (Fig EV3A-C). As shown in Fig EV3D, p13 knockdown did not induce autophagy, as determined by measuring the conversion of LC3-I to LC3-II, which is indicative of autophagic activity [34]. These results suggest that p13 knockdown prevents rotenone-induced mitochondrial dysfunction independently of PINK1associated autophagy. We found that a mitochondrial uncoupler, carbonyl cyanide m-chlorophenylhydrazone (CCCP), but not rotenone, significantly increased PINK1 accumulation (Fig EV3A-C). The underlying mechanism of these results remains unclear, but we found that rotenone mildly reduced $\Delta \Psi \mathrm{m}$ compared to CCCP (Fig EV3E). Since PINK1 accumulates on the mitochondrial outer membrane following the loss of $\Delta \Psi \mathrm{m}$ [19], the rotenone-induced depolarization of $\Delta \Psi \mathrm{m}$ may not be sufficient to trigger PINK1 accumulation. We also found that the CCCP-induced reduction in $\Delta \Psi \mathrm{m}$ was slightly reversed in p13-knockdown cells (Fig EV3F), which is likely to cause the slight decrease in PINK1 accumulation as compared to that in the controlknockdown cells (Fig EV3A). In addition, we found that CCCP treatment did not decrease p13 mRNA levels (Fig EV3G) and that the CCCP-induced decrease in p13 levels was prevented by bafilomycin A1, an autophagy inhibitor (Fig EV3H), suggesting that p13 may be partially degraded by CCCP-mediated autophagy ahead of the other mitochondrial proteins such as Tim23 and Hsp60 (Fig EV3B and C).

\section{Neuronal p13 expression in parkinsonian toxin exposure and PD}

We next investigated the in vivo function of p13 using an acute MPTPinduced experimental PD model in which nigrostriatal dopaminergic neurons are selectively damaged [15]. Based on an in situ hybridization analysis, p13 mRNA was widely expressed in different brain regions, including the olfactory bulb, cortex, hippocampus, cerebellum, striatum and midbrain (Fig EV4A). Western blot analysis indicated that endogenous p13 protein was highly expressed in specific regions, including the cortex, thalamus, midbrain and cerebellum, in mice (Fig EV4C). Using the MPTP-induced PD model, we next examined whether MPTP application changes $p 13$ expression levels in the various regions of the brain. We found that MPTP significantly decreased p13 mRNA expression levels selectively in the midbrain among the examined samples (Fig EV4D). We also found that p13 expression in the midbrain of MPTP-treated mice was decreased compared to that of vehicle-treated mice (Fig EV4E). These data are 
A
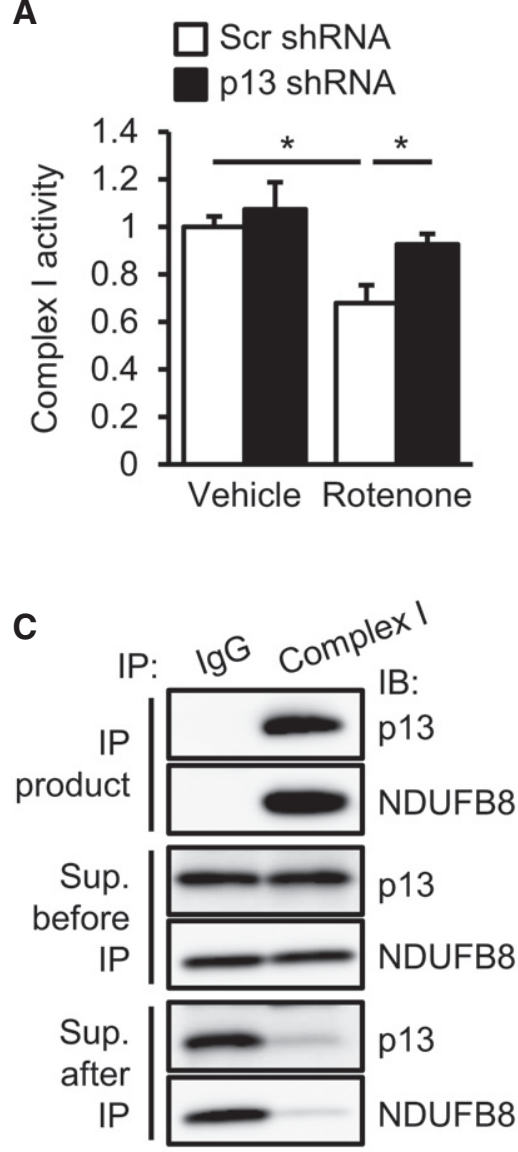
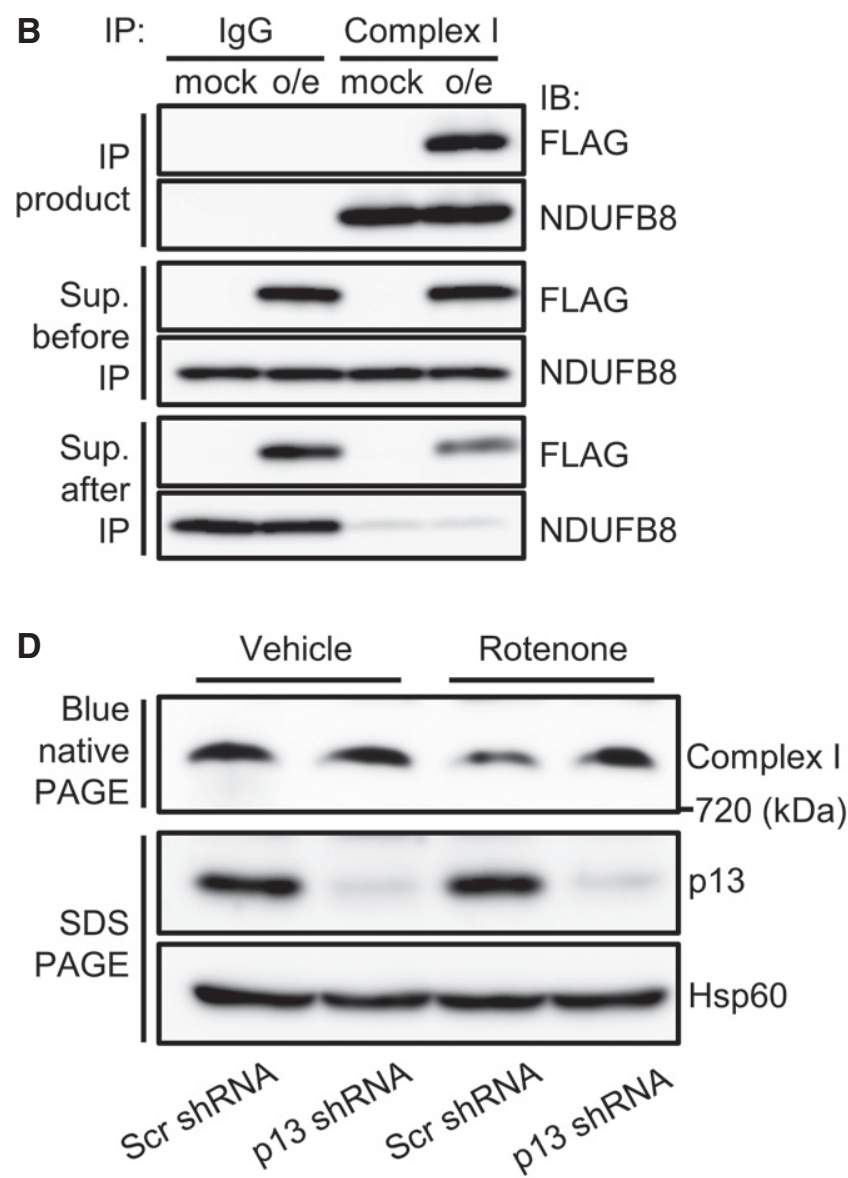

Figure 3. p13 knockdown maintains complex I activity in rotenone-treated SH-SY5Y cells.

A Prevention of the rotenone-induced decrease in complex I activity in p13 shRNA-infected cells. Complex I activity was measured on the basis of NADH-oxidizing activity. Data are presented as the mean $\pm \operatorname{SEM}(n=3)$. ${ }^{*} P<0.05$ by the Tukey-Kramer test.

B, C Physical interaction between overexpressed p13 (B) or endogenous p13 (C) and complex I proteins. Lysates were immunoprecipitated with anti-complex I antibody and control IgG. The lysates and immunoprecipitates were subjected to Western blotting with antibodies against FLAG (B), p13 (C) or NDUFB8 (complex I protein). Cells were infected with lentiviral vectors expressing mock or FLAG-tagged p13 (p13 o/e, B).

D Prevention of rotenone-induced impairment of complex I assembly by p13 knockdown in p13 shRNA-infected cells. Mitochondrial fractions were subjected to blue native PAGE, followed by Western blotting with an antibody against NDUFB8 (complex I protein). Levels of p13 and Hsp60 (for loading control) in the mitochondrial fraction were analysed.

Data information: Seventy-two hours after infection with lentiviral vectors expressing scrambled shRNA (Scr shRNA) or p13 shRNA, cells were stimulated with vehicle or $100 \mathrm{nM}$ rotenone for $24 \mathrm{~h}(\mathrm{~A}, \mathrm{D})$.

consistent with the vulnerability of midbrain dopaminergic neurons to MPTP [15]. Likewise, we observed that rotenone and $\mathrm{MPP}^{+}$ decreased the expression of p13 mRNA in SH-SY5Y cells (Fig EV5A and $\mathrm{B}$ ). While $\mathrm{MPP}^{+}$also decreased the p13 protein levels as well as p13 mRNA levels, rotenone treatment did not decrease p13 protein levels (Fig EV5C and D). The reason for the discrepancy is currently unclear. One possibility is that rotenone may inhibit p13 protein degradation through impairment of lysosomal functions [35]. These results indicate that p13 expression in dopaminergic neurons is decreased by parkinsonian toxicants and suggest that p13 reduction might function as part of an endogenous protective mechanism against PD pathogenesis. Alternatively, many mitochondria proteins are downregulated under toxin-induced conditions [36]; thus, the downregulation of p13 may be a downstream effect of mitochondrial dysfunction. In addition, $p 13$ mRNA expression was not decreased by rotenone at early time points ( 2 and $6 \mathrm{~h}$ ), when the mitochondrial dysfunction determined by the reduction in TMRE intensity was already marked (Fig EV5E and F).

\section{p13 heterozygous knockout prevents MPTP-induced motor deficits and neurodegeneration in mice}

To examine the effects of the suppression of p13 expression in an in vivo $\mathrm{PD}$ model, we generated $p 13$ knockout mice using the CRISPR/Cas9 system (Fig EV6A-D). We used $p 13^{+/-}$mice to evaluate the in vivo effects of the p13 knockdown because $p 13^{-/-}$mice showed high lethality shortly after birth (Fig EV6E). We first confirmed that the acute MPTP administration to $p 13^{+/+}$mice caused a marked deficit in motor coordination as measured by rotarod performance (Fig 4A). In sharp contrast to $p 13^{+/+}$mice, $p 13^{+/-}$mice did 

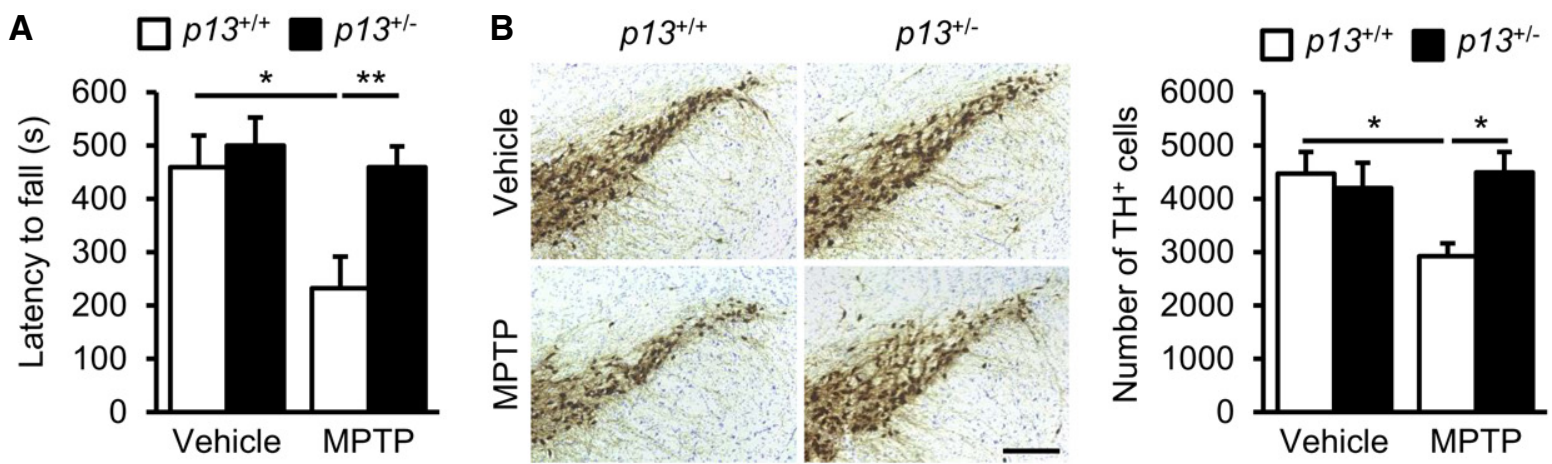
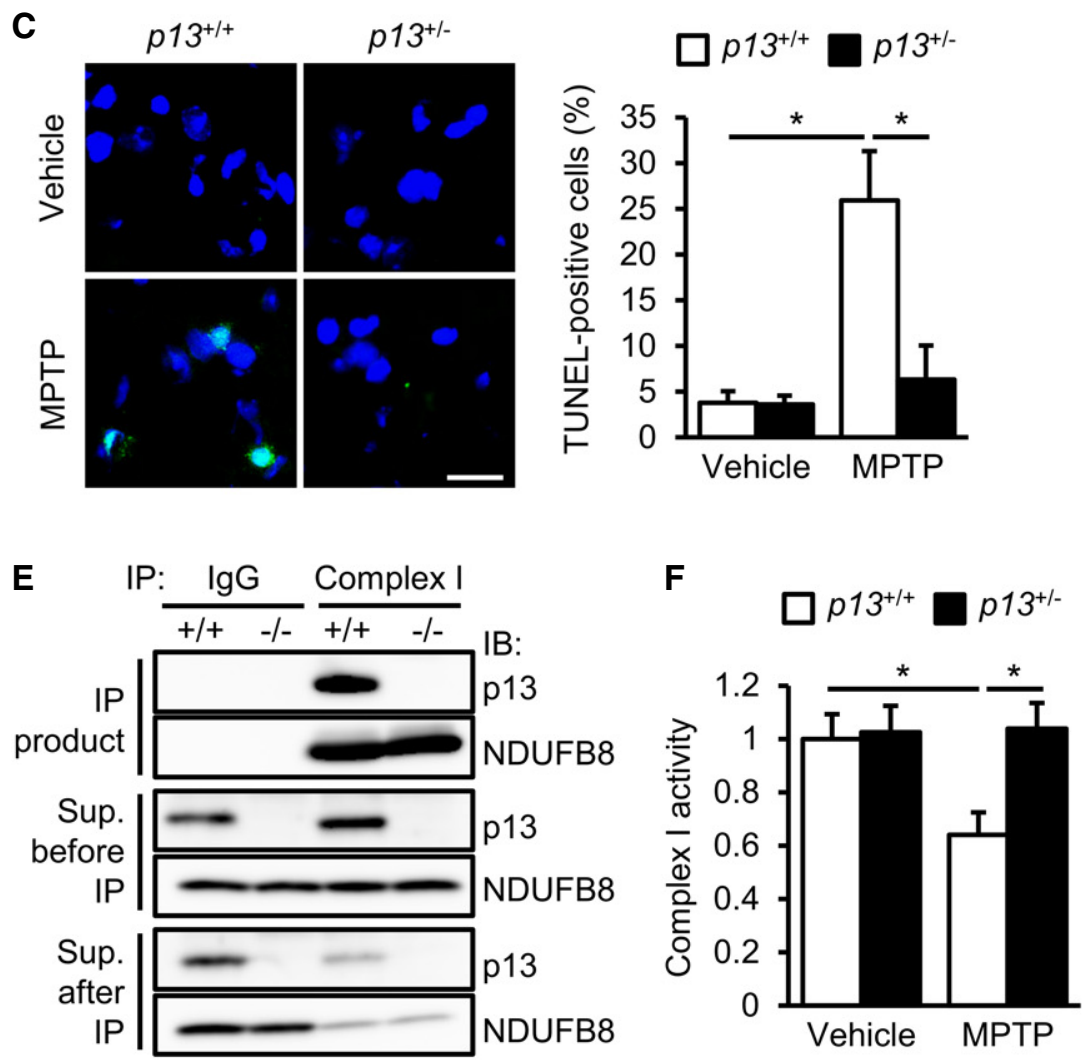

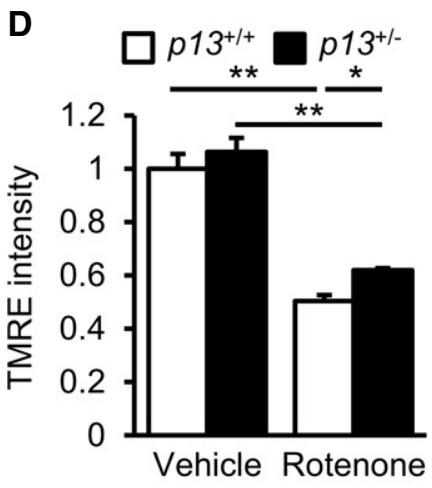

G

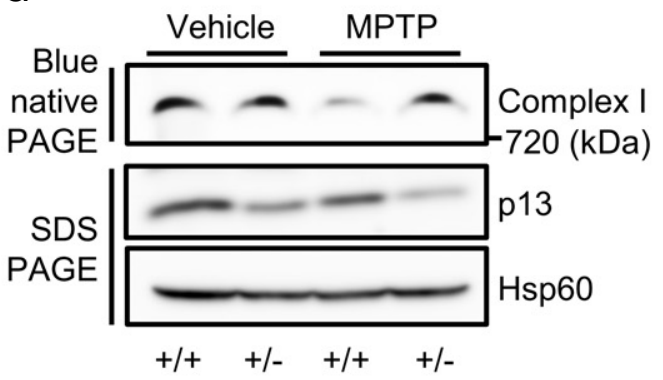

Figure 4. $p 13$ heterozygous knockout prevents MPTP-induced behavioural deficits and neurodegeneration in mice.

A Prevention of MPTP-induced motor deficits on the rotarod test in $p 13^{+/-}$mice. Latency to fall in two consecutive trials is presented ( $\left.n=10\right)$.

B Prevention of MPTP-induced decrease in $\mathrm{TH}^{+}$neurons in the substantia nigra of $p 13^{+/-}$mice analysed by immunohistochemistry $(n=4)$. Representative images (left) and the quantification of the number of $\mathrm{TH}^{+}$cells in the substantia nigra (right). Scale bar, $200 \mu \mathrm{m}$.

C Prevention of MPTP-induced increase in TUNEL-positive cells in the substantia nigra of $p 13^{+/-}$mice $(n=3)$. Representative images (left, TUNEL, shown in green; Hoechst, shown in blue) and the quantification of percentage of TUNEL-positive cells (right). Scale bar, $20 \mu \mathrm{m}$.

D Attenuation of rotenone-induced decrease in $\Delta \Psi \mathrm{m}$ in isolated mitochondria from the midbrain of $p 13^{+/-}$mice $(n=3)$. Isolated mitochondria were exposed to $1 \mu \mathrm{M}$ rotenone for $10 \mathrm{~min}$.

E Physical interaction between endogenous p13 and complex I proteins in the brain. Brain extracts from $p 13^{+/+}$and $p 13^{-1-}$ mice were immunoprecipitated with anticomplex I antibody and control IgG. The lysates and immunoprecipitates were subjected to Western blotting with antibodies against p13 and NDUFB8 (complex I protein).

F Prevention of the MPTP-induced decrease in complex I activity in the midbrain of $p 13^{+/-}$mice $(n=3)$. Complex I activity was measured on the basis of $\mathrm{NADH}-$ oxidizing activity.

G Prevention of MPTP-induced impairment of complex I assembly in the midbrain of $p 13^{+/-}$mice. Mitochondrial fractions were subjected to blue native PAGE, followed by Western blotting with an antibody against NDUFB8 (complex I protein). Levels of p13 and Hsp60 (for loading control) in the mitochondrial fraction were analysed. Data information: All data are presented as the mean \pm SEM. ${ }^{*} P<0.05,{ }^{* \star} P<0.01$ by the Tukey-Kramer test. 
not display MPTP-induced motor deficits (Fig 4A). We then examined the MPTP-induced degeneration of dopaminergic neurons in the substantia nigra of $p 13^{+/+}$and $p 13^{+/-}$mice by using Stereo Investigator software with a fractionator (MBF Bioscience). We found that the MPTP-induced reduction in the number of tyrosine hydroxylase-expressing $\left(\mathrm{TH}^{+}\right)$cells was almost completely reversed in $p 13^{+/-}$mice compared with $p 13^{+/+}$mice (Fig 4B). In vehicletreated mice, there was no difference in the number of $\mathrm{TH}^{+}$cells between $\mathrm{p}^{+3^{++}}$and $\mathrm{p} 13^{+/-}$mice (Fig 4B). Furthermore, we measured the optical density of $\mathrm{TH}^{+}$fibres in the striatum and found that MPTP-induced reduction in the optical density of $\mathrm{TH}^{+}$ fibres was not reversed in $p 13^{+/-}$mice compared with $p 13^{+/+}$mice (Fig EV6F). As previously reported [37], these results suggest that the restoration of dopaminergic function in the substantia nigra is important for the improvement in MPTP-induced motor dysfunction. Furthermore, we found that the percentage of TUNEL-positive cells was decreased in the substantia nigra of MPTP-treated $p 13^{+/-}$ mice (Fig 4C). We also observed that the conversion of LC3 was not induced in the mouse substantia nigra by MPTP regardless of the difference of p13 expression (Fig EV6G). Interestingly, we found that the rotenone-induced decrease in $\Delta \Psi \mathrm{m}$ was slightly attenuated in isolated mitochondria from the midbrain of $\mathrm{p} 3^{+/-}$mice (Fig 4D). We found that the endogenous p13 co-precipitated with complex I proteins in the brain samples (Fig 4E). The activity and assembly of mitochondrial complex I in the midbrain of $p 13^{+/+}$ mice were impaired by MPTP, whereas those processes were unimpaired in $p 13^{+/-}$mice (Fig $4 \mathrm{~F}$ and G). These results argue that the targeted knockdown of p13 protects against experimental parkinsonism in vivo as well as in vitro. In addition, the complex I protein level in the brain of $p 13^{-1-}$ mice was virtually identical to that of $p 13^{+/+}$mice (Fig EV6H).

$\mathrm{PD}$ is characterized by the progressive and selective degeneration of nigrostriatal dopaminergic neurons, but there is no established neuroprotective or neurorestorative treatment for PD. We demonstrated for the first time that p13 expression was reduced by parkinsonian toxicants both in cell culture and in the mouse midbrain (Figs EV4D and E, and EV5A, B and D). Our mechanistic studies show that p13 knockdown prevented parkinsonian toxicantinduced mitochondrial dysfunction and cell death by enhancing complex I activity (Figs 2C-E and 3A). p13 heterozygous knockout also significantly protected mice against MPTP-induced motor deficits and nigral dopaminergic neurodegeneration (Fig 4). Thus, these results suggest that the reduction in p13 expression can act as a protective factor against PD-related pathogenesis and that the manipulation of p13 expression might be a novel and beneficial treatment option for PD.

Controlling mitochondrial integrity has been regarded as a convincing strategy for PD therapeutics $[8,9,38]$. Indeed, reducing excessive mitochondrial oxidative stress [22,39], stimulating PINK1/Parkin-mediated autophagy $[20,40]$ and promoting mitochondrial biogenesis $[41,42]$ have shown beneficial effects in PD models. However, understanding the PD pathogenesis associated with mitochondria continues to be a major research challenge because mitochondrial function is regulated by a complex network. Our findings suggest that 13 is a novel player in maintaining mitochondrial integrity. Using cell culture and mouse models, our study demonstrates that the reduction in p13 protects dopaminergic cells from mitochondrial dysfunction. Further analyses of the roles of p13 in conjunction with other mitochondrial proteins are important for clarifying the p13-mediated regulation of mitochondrial function in PD pathogenesis. To evaluate the role of p13 in PD pathogenesis, we examined the expression level of p13 mRNA in the postmortem brain tissue of three PD patients and three age-matched control subjects. p13 mRNA expression levels in the frontal cortex of the patients with PD tended to be lower than those of the control subjects (data not shown); however, the result is necessarily preliminary, mainly owing to the small sample size. The additional therapeutic potential of p13 reduction, such as lack of tolerance or prevention of the disease progression, would be evaluated via further comparative studies using PD patient samples.

On the basis of the present data, the most likely mechanism for the protective effects of p13 reduction against PD pathogenesis is the regulation of complex I activity. p13 has an LYR motif that is associated with the assembly of complex I [43]. We found that p13 physically interacts with complex I proteins (Figs $3 \mathrm{~B}$ and $\mathrm{C}$, and $4 \mathrm{E})$. A recent study also demonstrated that p13 is likely to interact with NDUFAB1, a subunit of complex I [24]. Thus, it is possible that p13 functions as an assembly factor of complex I. In this study, p13 reduction prevented the decrease in complex I activity and assembly by parkinsonian toxicants (Figs $3 \mathrm{~A}$ and $\mathrm{D}$, and $4 \mathrm{~F}$ and $\mathrm{G}$ ). One possible mechanism is that p13 reduction decreases the sensitivity of complex I to parkinsonian toxicants. It remains unclear why 80 $90 \%$ knockdown of p13 in SH-SY5Y cells had no effect on complex I activity under basal conditions. We found that, in contrast to the heart, there were no significant differences in complex I activity in the brain among $p 13^{-/-}, p 13^{+/-}$and $p 13^{+/+}$mice (Fig EV6I and $\mathrm{J})$. These results suggest that the importance of p13 function may differ between cell types.

The decreased expression levels of $p 13$ mRNA caused by parkinsonian toxicants were comparable to those of p13 shRNA; however, shRNA-mediated p13 knockdown but not parkinsonian toxicantmediated decreased p13 expression protected mitochondrial function against these stresses. The response to decreased $p 13$ expression by parkinsonian toxicants may be too late to exert protective effects. Indeed, rotenone rapidly decreased $\Delta \Psi \mathrm{m} 2 \mathrm{~h}$ after treatment (Fig EV5E), but did not alter p13 mRNA levels under the same conditions (Fig EV5F). Considering that mitochondria change their functions early upon stimulation and then promote neurodegeneration, performing p13 knockdown in advance is suggested to be necessary to realize the protective effects of p13 reduction in preventing PD.

In conclusion, we demonstrated that the targeted knockdown of the new mitochondrial protein p13 prevents mitochondrial dysfunction and dopaminergic neuronal death in both in vitro and in vivo PD models. Our findings should help to explain the molecular pathogenesis of PD. Further studies are needed to propose a new PD therapeutic that targets p13.

\section{Materials and Methods}

\section{Cell culture}

Human neuroblastoma SH-SY5Y cells were cultured in Dulbecco's modified Eagle's medium (Nissui, Tokyo, Japan) supplemented with $10 \%$ foetal bovine serum and $4 \mathrm{mM}$ L-glutamine at $37^{\circ} \mathrm{C}$ in a 
humidified atmosphere of $5 \% \mathrm{CO}_{2}$. Lenti-X $293 \mathrm{~T}$ cells (Clontech, Mountain View, CA, USA) were maintained in Dulbecco's modified Eagle's medium for lentivirus production.

\section{Lentiviral vectors}

Recombinant lentivirus was prepared as described previously [44]. To generate lentiviral vectors expressing mouse p13, mouse cDNA (NP_079639) was amplified via PCR and subcloned into the plasmid CSII-EF-MCS (a generous gift from Dr. Kazuki Nagayasu at Kyoto University). For knockdown experiments, we used a MISSION shRNA construct targeting human p13 (TRCN0000163507, SigmaAldrich, St Louis, MO, USA), a non-targeted shRNA control (SHC002, Sigma-Aldrich), another shRNA (p13 shRNA \#2) targeting another sequence of p13 (TRCN0000159440, Sigma-Aldrich) and an shRNA construct targeting human PINK1 (TRCN0000199193, Sigma-Aldrich). For rescue experiments, mouse p13 cDNA was subcloned into the MISSION shRNA construct. SH-SY5Y cells were infected with lentiviral vectors at a multiplicity of infection of approximately 20 for overexpression experiments or 40 for knockdown experiments.

\section{Immunocytochemistry}

Cells were fixed for 15 min in $4 \%$ paraformaldehyde in phosphatebuffered saline (PBS). Permeabilization was performed for $10 \mathrm{~min}$ in $0.2 \%$ Triton X-100. The cells were blocked for $1 \mathrm{~h}$ in $1 \%$ bovine serum albumin (BSA, Nacalai Tesque, Kyoto, Japan) in PBS. The cells were then probed with the primary antibodies against p13 (1:250, HPA045663, Atlas Antibodies, Stockholm, Sweden), Hsp60 (1:250, sc-1052, Santa Cruz Biotechnology, Santa Cruz, CA, USA) and complex I proteins (1:500, ab109798, Abcam, Cambridge, UK) overnight at $4^{\circ} \mathrm{C}$. Donkey anti-rabbit IgG Alexa Fluor 594 (1:1,000, A-21207, Thermo Fisher Scientific, Tokyo, Japan), donkey anti-goat IgG Alexa Fluor 488 (1:1,000, A-11055, Thermo Fisher Scientific) and donkey anti-mouse IgG Alexa Fluor 488 (1:1,000, A-21202, Thermo Fisher Scientific) antibodies were used as secondary antibodies. Images were acquired with a confocal microscope (FV1000D, Olympus, Tokyo, Japan).

\section{Mitochondrial toxins}

Parkinsonian toxicants rotenone, $\mathrm{MPP}^{+}, \mathrm{MPTP}$ and mitochondrial uncoupler CCCP were purchased from Sigma-Aldrich.

\section{Flow cytometry}

Cells were incubated with Opti-MEM ${ }^{\circledR}$ (Thermo Fisher Scientific) containing $200 \mathrm{nM}$ TMRE (Thermo Fisher Scientific) or $200 \mathrm{nM}$ MitoTracker Green FM (Thermo Fisher Scientific) for $20 \mathrm{~min}$ at $37^{\circ} \mathrm{C}$. After trypsinization, cells were resuspended in PBS containing $0.2 \% \mathrm{BSA}$, and fluorescence was then detected using a BD Accuri ${ }^{\mathrm{TM}}$ C6 Flow Cytometer (BD Biosciences, San Jose, CA, USA).

\section{Western blotting}

Protein samples were resolved by SDS-PAGE and transferred to polyvinylidene fluoride membranes (Millipore, Darmstadt,
Germany). After being blocked with 5\% BSA in Tris-buffered saline (TBS) containing $0.1 \%$ Tween 20 , the membranes were probed with primary antibodies against p13 (1:250), cleaved PARP (1:1,000, 5625S, Cell Signaling, Danvers, MA, USA), $\beta$-actin $(1: 4,000$, MAB1501, Chemicon, Temecula, CA, USA), Hsp60 (1:250), Lamin A/C (1:1,000, 2032S, Cell Signaling), Tim23 (1:1,000, 611223, BD Biosciences), GAPDH (1:5,000, 2118S, Cell Signaling), Tom20 (1:1,000, 13929S, Cell Signaling), FLAG $(1: 1,000,2368$, Cell Signaling), NDUFB8 (1:2,000, ab110242, Abcam), PINK1 (1:1,000, 6946S, Cell Signaling), LC3 (1:1,000, NB100-2220, Novus Biologicals, Littleton, CO, USA) or NDUFS4 (1:1,000, EPR7831, Abcam) overnight at $4^{\circ} \mathrm{C}$. After incubation with the horseradish peroxidase-conjugated secondary antibodies against rabbit IgG $(1: 2,000,55689$, Cappel, Cochranville, PA, USA), mouse IgG (1:2,000, 55563, Cappel) and goat IgG $(1: 2,000,55358$, Cappel) for $1 \mathrm{~h}$ at room temperature, proteins were detected by chemiluminescence (ImmunoStar Zeta, Wako, Osaka, Japan). Data acquisition and analysis were performed using an LAS 4000 image analyser (GE Healthcare, Piscataway, NJ, USA).

\section{TUNEL staining}

TUNEL staining was performed using an In Situ Cell Death Detection Kit (Roche Diagnostics, Basel, Switzerland) according to the manufacturer's instruction. Cells were further counterstained with Hoechst. To evaluate the extent of apoptosis, we counted TUNELpositive cells in five fields per sample for number of TUNELpositive cells.

\section{Subcellular fractionation and mitochondrial isolation}

Subcellular fractionation was performed using FOCUS ${ }^{\mathrm{TM}}$ SubCell Kit (G-Biosciences, Lt. Louis, MO, USA) according to the manufacturer' instruction. Each fraction was determined by Western blotting using the following antibodies: Lamin A/C for nuclear, Tim23 for mitochondrial and GAPDH for cytoplasmic fractions.

For digitonin treatment, blue native PAGE and TMRE staining, mitochondria were isolated according to a previously reported method [45]. Briefly, cells or tissues were homogenized by a Dounce homogenizer in ice-cold IBc buffer (10 mM Tris-MOPS, $1 \mathrm{mM}$ EGTA-Tris, $0.2 \mathrm{M}$ sucrose, $\mathrm{pH}$ 7.4). The homogenate was centrifuged at $600 \mathrm{~g}$ at $4^{\circ} \mathrm{C}$ for $10 \mathrm{~min}$, and the supernatant was again centrifuged at $7,000 \mathrm{~g}$ at $4^{\circ} \mathrm{C}$ for $10 \mathrm{~min}$. The precipitated pellets were washed once with IBc buffer. Protein concentration was determined with a BCA Protein Assay Kit (Thermo Fisher Scientific).

\section{Digitonin treatment of isolated mitochondria}

Isolated mitochondria were treated with $20 \mu \mathrm{g} / \mathrm{ml}$ proteinase $\mathrm{K}$ in the absence or presence of ascending concentrations of digitonin $(0.01,0.05$ and $0.1 \%)$ for $10 \mathrm{~min}$, and then, the protease reaction was terminated by the addition of $5 \mathrm{mM}$ phenylmethylsulphonyl fluoride. The mitochondrial extracts were subjected to Western blotting using the following antibodies: p13, Tom20 for the outer mitochondrial membrane, Tim23 for the inner mitochondrial membrane and Hsp60 for the mitochondrial matrix 


\section{Complex I activity assay}

Complex I activity was assayed using a complex I enzyme activity microplate assay kit (Abcam). Cell pellets or brain tissues were lysed according to the manufacturer's protocol. A measure of $250 \mu \mathrm{g}$ protein of each sample was added to each well of the microplate that was pre-coated with anti-complex I antibody, and the microplate was incubated for $3 \mathrm{~h}$ at room temperature. After the incubation, complex I activity was determined by monitoring the oxidation of $\mathrm{NADH}$ to $\mathrm{NAD}^{+}$, coupled to the reporter dye, which leads to increased absorbance at $450 \mathrm{~nm}$. Absorbance was measured by an iMark microplate reader (Bio-Rad, Hercules, CA, USA). The activity was expressed as the change in absorbance per minute.

\section{Co-immunoprecipitation}

For the detection of binding between p13 and complex I proteins, SH-SY5Y cells and brains were lysed with a buffer containing 50 mM Tris- $\mathrm{HCl}$ (pH 8.0), $100 \mathrm{mM} \mathrm{NaCl}, 5$ mM EDTA, 1\% NP-40 and $10 \mathrm{mM} \mathrm{NaF}$. Lysates were incubated with an anti-complex I immunocapture antibody (1:500, Abcam) and protein G-Sepharose (GE Healthcare) overnight at $4^{\circ} \mathrm{C}$. Immunocomplexes were washed five times with the buffer, separated by SDS-PAGE and subjected to Western blotting.

\section{Analysis of complex I assembly by blue native PAGE}

Mitochondrial proteins $(150 \mu \mathrm{g})$ were separated on a $3-12 \%$ gradient blue native PAGE gel according to the method previously reported [46]. After electrophoresis, the complexes were subjected to Western blotting with an antibody against NDUFB8 for complex I.

\section{Animals}

C57BL/6J mice (SLC, Hamamatsu, Japan) were used in this study. Mice were maintained on a 12-h light-dark cycle (lights on at 8:00 a.m.) at a controlled room temperature $\left(22 \pm 1^{\circ} \mathrm{C}\right)$. Water and food (CMF, Oriental Yeast, Osaka, Japan) were available ad libitum. All animal care and handling procedures were approved by the Animal Care and Use Committee of Osaka University. All efforts were made to minimize the number of animals used.

\section{MPTP administration and rotarod test}

Mice were subcutaneously administered with MPTP $(10 \mathrm{mg} / \mathrm{kg})$ four times at 2-h intervals. Motor performance was assessed by the rotarod test $24 \mathrm{~h}$ after the first administration of MPTP, as described previously $[37,47,48]$. The rotarod (Neuroscience Inc., Tokyo, Japan) consisted of a rotating rod $(2.8 \mathrm{~cm}$ diameter $)$ and individual compartments for each mouse. Briefly, mice were trained for 3 days prior to MPTP administration in an acceleration mode (2-16 rpm) for over $120 \mathrm{~s}$. The training was repeated at a fixed speed (16 rpm) until the mice were able to stay on the rod for at least $600 \mathrm{~s}$. Motor coordination was measured at a speed of $16 \mathrm{rpm}$ for a maximum recording time of $600 \mathrm{~s}$. Mice were tried twice on 1 day with an interval time of $30 \mathrm{~min}$, and average latency to fall was measured.

\section{Immunohistochemistry}

Mice were sacrificed $72 \mathrm{~h}$ after the first MPTP administration. Tissue preparation was performed as described previously [49]. Postfixed mouse brains were cut into $20-\mu \mathrm{m}$ sections containing the substantia nigra ( -3.00 to $-3.26 \mathrm{~mm}$ with respect to bregma) or the striatum $(+0.80$ through $+0.54 \mathrm{~mm}$ with respect to bregma) using a cryostat (Leica Biosystems, Tokyo, Japan). Sections were incubated for $30 \mathrm{~min}$ in $0.3 \%$ hydrogen peroxide in PBS and then blocked with $1 \%$ normal goat serum (Vectastain ABC HRP kit; Vector Laboratories, Burlingame, CA, USA) in PBS for $1 \mathrm{~h}$ at room temperature. The sections were incubated with the antibody against $\mathrm{TH}(1: 1,000$, AB152, Millipore) in PBS containing 1\% normal goat serum and $0.1 \%$ Triton X-100 overnight at $4^{\circ} \mathrm{C}$. Subsequently, sections were incubated in a secondary antibody solution containing biotinylated anti-rabbit IgG (1:125, Vectastain ABC HRP kit; Vector Laboratories) in $\mathrm{PBS}$ for $30 \mathrm{~min}$ at room temperature and then reacted with the avidin-biotin peroxidase complex (1:125, Vectastain ABC HRP kit; Vector Laboratories) in PBS for $30 \mathrm{~min}$ at room temperature. Visualization was performed using a DAB substrate kit (Nichirei Corporation, Tokyo, Japan) according to the manufacturer's protocol. Penetration was conducted with ethanol (95 and 100\%) and xylene. Sections were also counterstained by Nissl staining. Images were acquired with a microscope (BZ-9000, Keyence, Osaka, Japan). The total number of $\mathrm{TH}^{+}$neurons in every fourth section of the substantia nigra was counted stereologically with Stereo Investigator software (MBF Bioscience, Williston, VT, USA) using the fractionator method.

\section{TMRE staining in isolated mitochondria from mice}

Isolated mitochondria from the mouse midbrain were stained with TMRE as described previously [50]. Briefly, isolated mitochondria $(75 \mu \mathrm{g})$ were incubated with $\mathrm{EB}$ buffer $(125 \mathrm{mM} \mathrm{KCl}, 10 \mathrm{mM}$ Tris-MOPS, $5 \mathrm{mM}$ glutamate, $2.5 \mathrm{mM}$ malate, $1 \mathrm{mM} \mathrm{K}$ phosphate and $10 \mathrm{mM}$ EGTA-Tris, pH 7.4) containing $1 \mu \mathrm{M}$ TMRE for $10 \mathrm{~min}$ at room temperature. The homogenate was centrifuged at $10,000 \mathrm{~g}$ at $4^{\circ} \mathrm{C}$ for $5 \mathrm{~min}$. The precipitated pellets were resuspended with the EB buffer, and fluorescence was then detected using a Spectramax M5e (Molecular Devices, Sunnyvale, CA, USA).

\section{Real-time RT-PCR}

Total RNA was isolated using QIAzol Lysis Reagent (QIAGEN, Tokyo, Japan) according to the manufacturer's protocol. Reverse transcription of total RNA $(1 \mu \mathrm{g})$ and real-time RT-PCR were performed as described previously [51]. Real-time RT-PCR was conducted with GoTaq qPCR Master Mix (Promega, Madison, WI, USA). The primer sequences were as follows:

Human p13_forward, 5'-AAGGCTTTCCGTGCACATCGG-3' Human p13_reverse, 5'-CAGCCCTTCCCTCCAGGCTGAT-3' Human $\beta$-actin_forward, 5'-CATGTGCAAGGCCGGCTTCG- $3^{\prime}$ Human $\beta$-actin_reverse, $5^{\prime}$-CTGGGTCATCTTCTCGCGGT-3' Human PINK1_forward, 5'-GCCATCAAGATGATGTGGAAC-3' Human PINK1_forward, 5'-GACCAGCTCCTGGCTCATT-3' Human ND1_forward, 5'-CACCTCTAGCCTAGCCGTTT-3' Human ND1_reverse, 5'-CCGATCAGGGCGTAGTTTGA-3' 
Human ND2_forward, 5'-CTTAAACTCCAGCACCACGAC-3' Human ND2_reverse, 5'-AGCTTGTTTCAGGTGCGAGA-3' Human ND3_forward, $5^{\prime}$-CCGCGTCCCTTTCTCCATAA-3' Human ND3_reverse, 5' -AGGGCTCATGGTAGGGGTAA-3' Human ND4_forward, 5' -ACAACACAATGGGGCTCACT-3' Human ND4_reverse, 5'-CCGGTAATGATGTCGGGGTT-3' Human ND4L_forward, 5' -TCGCTCACACCTCATATCCTC-3' Human ND4L_reverse, 5'-AGGCGGCAAAGACTAGTATGG-3' Human ND5_forward, 5'-TCCATTGTCGCATCCACCTT-3' Human ND5_reverse, 5'-GGTTGTTTGGGTTGTGGCTC-3' Human ND6_forward, 5'-GGGTTGAGGTCTTGGTGAGT-3' Human ND6_reverse, 5'-ACCAATCCTACCTCCATCGC-3' Human CYTB_forward, 5'-TCTTGCACGAAACGGGATCA- $3^{\prime}$ Human CYTB_reverse, $5^{\prime}$-CGAGGGCGTCTTTGATTGTG-3' Human COX1_forward, 5'-TCCTTATTCGAGCCGAGCTG-3' Human COX1_reverse, 5' -ACAAATGCATGGGCTGTGAC- $3^{\prime}$ Human COX2_forward, 5'-AACCAAACCACTTTCACCGC-3' Human COX2_reverse, 5' -CGATGGGCATGAAACTGTGG-3' Human ATP6_forward, 5'-TTCGCTTCATTCATTGCCCC-3' Human ATP6_reverse, 5'-GGGTGGTGATTAGTCGGTTGT-3' Human ATP8_forward, 5'-ACTACCACCTACCTCCCTCAC-3' Human ATP8_reverse, 5'-GGCAATGAATGAAGCGAACAGA-3' Mouse p13_forward, 5'-AAGGCTTTCCGTGCACATCGG-3' Mouse p13_reverse, 5'-CAGCCCTTCCCTCCAGGCTGAC-3' Mouse $\beta$-actin_forward, $5^{\prime}$-ACCCACACTGTGCCCATCTA- $3^{\prime}$ Mouse $\beta$-actin_reverse, $5^{\prime}$-GCCACAGGATTCCATACCCA- $3^{\prime}$

\section{LDH assay}

LDH levels released from damaged cells were measured using a cytotoxicity detection kit (Roche Diagnostics) according to the manufacturer's protocol. The culture medium of the cells was incubated with the reaction mixture at room temperature for $30 \mathrm{~min}$. Signals at wavelengths of 490 and $620 \mathrm{~nm}$ were measured by spectrophotometry.

\section{In situ hybridization}

In situ hybridization analysis was performed on sagittal brain sections as described previously [52]. Complementary DNA fragments encoding mouse p13 cDNA (NP_079639) were used as templates to synthesize $\left[{ }^{35} \mathrm{~S}\right]$ CTP-labelled cRNA probes.

\section{CRISPR/Cas9-based knockout of p13 in mice}

p13 knockout mice were generated using the CRISPR/Cas9 system. The sgRNA sequences 5'-GACAGAAAAATGGCGGCCCT-3' and $5^{\prime}$-CGCTACCTGAACGCGGCCAC-3' targeting exon 1 of the mouse p13 gene were cloned into pX330 (Addgene, Cambridge, MA, USA). pCAG-EGxxFP was used to examine the efficiency of the target DNA cleavage by the gRNAs and Cas9 [53]. The pX330 plasmids containing each sgRNA sequence were injected into the pronuclear stage eggs. Mice that harboured a 71-bp deletion in exon 1 of the $p 13$ gene were obtained. The genotypes of all mice were analysed by PCR for a mutated p13 locus using the following primers:

Genotyping primer p13_forward, 5'-CACCTTCCCTTGCTCTCCTG-3' Genotyping primer p13_reverse, $5^{\prime}$-GAGACCCTCTATCACCTGCG- $3^{\prime}$

\section{Data analysis and statistics}

Statistical analysis was performed using Statview (SAS Institute Japan Ltd., Tokyo, Japan), and significant differences were determined by Student's $t$-test, Dunnett's test and the Tukey-Kramer test. The threshold for statistical significance was defined as $P<0.05$.

Expanded View for this article is available online.

\section{Acknowledgements}

We thank NPO Biotechnology Research and Development for technical assistance. We are grateful to Dr. Koji Okamoto (Osaka University) for helpful discussions. This work was supported in part by the Japan Society for the Promotion of Science (JSPS) KAKENHI grant numbers JP25670038 (NS), JP17H03989 (HH), JP17K19488 (HH) and JP26293020 (HH); Project MEET, Osaka University Graduate School of Medicine (NS); Mitsubishi Tanabe Pharma Corporation (NS); the JSPS Program for Advancing Strategic International Networks to Accelerate the Circulation of Talented Researchers, grant number S2603 (HH); the SRPBS and Brain/MINDS from AMED (HH); JSPS Research Fellowships for Young Scientists, grant number JP15J06322 (NI); and grants for research from the Uehara Memorial Foundation, Japan (NS).

\section{Author contributions}

$\mathrm{NI}$, SO, KI, SH and YS performed experiments. NI, SO and Al generated p13-deficient mice. KB, HM and HF assessed $p 13$ mRNA levels in autopsied brain tissues. NI, SO, AK, TN, YA, AH-T, KS, NS and HH analysed the data. AK, TN, HM, $Y A, A H-T, K S, N S$ and $H H$ provided guidance and/or senior supervision. NI, AK, TN, NS and HH wrote the manuscript. NI and SO prepared the figures under supervision from AK, TN, NS and HH. All authors provided input and corrections to the preparation of the manuscript and figures.

\section{Conflict of interest}

The authors declare that they have no conflict of interest.

\section{References}

1. Berg D, Postuma RB, Bloem B, Chan P, Dubois B, Gasser T, Goetz CG, Halliday GM, Hardy J, Lang AE et al (2014) Time to redefine PD? Introductory statement of the MDS Task Force on the definition of Parkinson's disease. Mou Disord 29: 454-462

2. Postuma RB, Berg D, Stern M, Poewe W, Olanow CW, Oertel W, Obeso J, Marek K, Litvan I, Lang AE et al (2015) MDS clinical diagnostic criteria for Parkinson's disease. Mou Disord 30: 1591-1601

3. Jenner P (2008) Molecular mechanisms of L-DOPA-induced dyskinesia. Nat Reu Neurosci 9: 665-677

4. Athauda D, Foltynie T (2015) The ongoing pursuit of neuroprotective therapies in Parkinson disease. Nat Reu Neurol 11: 25-40

5. Kalia LV, Kalia SK, Lang AE (2015) Disease-modifying strategies for Parkinson's disease. Mou Disord 30: 1442-1450

6. Choong CJ, Baba K, Mochizuki H (2016) Gene therapy for neurological disorders. Expert Opin Biol Ther 16: 143-159

7. Ryan BJ, Hoek S, Fon EA, Wade-Martins R (2015) Mitochondrial dysfunction and mitophagy in Parkinson's: from familial to sporadic disease. Trends Biochem Sci 40: 200-210

8. Schon EA, Przedborski S (2011) Mitochondria: the next (neurode)generation. Neuron 70: $1033-1053$ 
9. Exner N, Lutz AK, Haass C, Winklhofer KF (2012) Mitochondrial dysfunction in Parkinson's disease: molecular mechanisms and pathophysiological consequences. EMBO J 31: 3038-3062

10. Choong CJ, Mochizuki H (2017) Gene therapy targeting mitochondrial pathway in Parkinson's disease. J Neural Transm 124: 193-207

11. Hattori N, Ikebe S, Tanaka M, Ozawa T, Mizuno Y (1993) Immunohistochemical studies on complexes I, II, III, and IV of mitochondria in Parkinson's disease. Adu Neurol 60: 292-296

12. Mizuno Y, Ohta S, Tanaka M, Takamiya S, Suzuki K, Sato T, Oya H, Ozawa T, Kagawa Y (1989) Deficiencies in complex I subunits of the respiratory chain in Parkinson's disease. Biochem Biophys Res Commun 163: 1450-1455

13. Schapira AH, Cooper JM, Dexter D, Clark JB, Jenner P, Marsden CD (1990) Mitochondrial complex I deficiency in Parkinson's disease. J Neurochem 54: $823-827$

14. Betarbet R, Sherer TB, Mackenzie G, Garcia-Osuna M, Panov AV, Greenamyre JT (2000) Chronic systemic pesticide exposure reproduces features of Parkinson's disease. Nat Neurosci 3: 1301-1306

15. Dauer W, Przedborski S (2003) Parkinson's disease: mechanisms and models. Neuron 39: 889-909

16. Porras G, Li Q, Bezard E (2012) Modeling Parkinson's disease in primates: the MPTP model. Cold Spring Harb Perspect Med 2: a009308

17. Manfredi G (2006) mtDNA clock runs out for dopaminergic neurons. Nat Genet 38: $507-508$

18. Schapira AH (2008) Mitochondria in the aetiology and pathogenesis of Parkinson's disease. Lancet Neurol 7: 97-109

19. Martinez-Vicente M (2017) Neuronal mitophagy in neurodegenerative diseases. Front Mol Neurosci 10: 64

20. Pickrell AM, Youle RJ (2015) The roles of PINK1, parkin, and mitochondrial fidelity in Parkinson's disease. Neuron 85: 257-273

21. Higashi S, Katagi K, Shintani N, Ikeda K, Sugimoto Y, Tsuchiya S, Inoue N, Tanaka S, Koumoto M, Kasai A et al (2015) p13 overexpression in pancreatic beta-cells ameliorates type 2 diabetes in high-fat-fed mice. Biochem Biophys Res Commun 461: 612-617

22. Lin MT, Beal MF (2006) Mitochondrial dysfunction and oxidative stress in neurodegenerative diseases. Nature 443: 787-795

23. Ott M, Gogvadze V, Orrenius S, Zhivotovsky B (2007) Mitochondria, oxidative stress and cell death. Apoptosis 12: 913-922

24. Floyd BJ, Wilkerson EM, Veling MT, Minogue CE, Xia C, Beebe ET, Wrobel $\mathrm{RL}$, Cho H, Kremer LS, Alston CL et al (2016) Mitochondrial protein interaction mapping identifies regulators of respiratory chain function. Mol Cell 63: 621-632

25. Han S, Udeshi ND, Deerinck TJ, Svinkina T, Ellisman MH, Carr SA, Ting AY (2017) Proximity biotinylation as a method for mapping proteins associated with mtDNA in living cells. Cell Chem Biol 24: 404-414

26. Galluzzi L, Blomgren K, Kroemer G (2009) Mitochondrial membrane permeabilization in neuronal injury. Nat Reu Neurosci 10: 481-494

27. Kroemer G, Galluzzi L, Brenner C (2007) Mitochondrial membrane permeabilization in cell death. Physiol Reu 87: 99-163

28. Yamano K, Matsuda N, Tanaka K (2016) The ubiquitin signal and autophagy: an orchestrated dance leading to mitochondrial degradation. EMBO Rep 17: $300-316$

29. Matsuda N, Sato S, Shiba K, Okatsu K, Saisho K, Gautier CA, Sou YS, Saiki S, Kawajiri S, Sato F et al (2010) PINK1 stabilized by mitochondrial depolarization recruits Parkin to damaged mitochondria and activates latent Parkin for mitophagy. J Cell Biol 189: 211-221

30. Narendra DP, Jin SM, Tanaka A, Suen DF, Gautier CA, Shen J, Cookson MR, Youle RJ (2010) PINK1 is selectively stabilized on impaired mitochondria to activate Parkin. PLOS Biol 8: e1000298
31. Kazlauskaite A, Martinez-Torres RJ, Wilkie S, Kumar A, Peltier J, Gonzalez A, Johnson C, Zhang J, Hope AG, Peggie M et al (2015) Binding to serine 65-phosphorylated ubiquitin primes Parkin for optimal PINK1-dependent phosphorylation and activation. EMBO Rep 16: 939-954

32. Narendra D, Tanaka A, Suen DF, Youle RJ (2008) Parkin is recruited selectively to impaired mitochondria and promotes their autophagy. J Cell Biol 183: 795-803

33. Twig G, Elorza A, Molina AJ, Mohamed H, Wikstrom JD, Walzer G, Stiles L, Haigh SE, Katz S, Las G et al (2008) Fission and selective fusion govern mitochondrial segregation and elimination by autophagy. EMBO J 27: $433-446$

34. Kabeya Y, Mizushima N, Ueno T, Yamamoto A, Kirisako T, Noda T, Kominami E, Ohsumi Y, Yoshimori T (2000) LC3, a mammalian homologue of yeast Apg8p, is localized in autophagosome membranes after processing. $E M B O$ J 19: $5720-5728$

35. Wu F, Xu HD, Guan JJ, Hou YS, Gu JH, Zhen XC, Qin ZH (2015) Rotenone impairs autophagic flux and lysosomal functions in Parkinson's disease. Neuroscience 284: 900-911

36. Zheng B, Liao Z, Locascio JJ, Lesniak KA, Roderick SS, Watt ML, Eklund AC, Zhang-James Y, Kim PD, Hauser MA et al (2010) PGC-1alpha, a potential therapeutic target for early intervention in Parkinson's disease. Sci Transl Med 2: 52ra73

37. Kawasaki T, Ishihara K, Ago Y, Baba A, Matsuda T (2007) Edaravone (3-methyl-1-phenyl-2-pyrazolin-5-one), a radical scavenger, prevents 1-methyl-4-phenyl-1,2,3,6-tetrahydropyridine-induced neurotoxicity in the substantia nigra but not the striatum.J Pharmacol Exp Ther 322: $274-281$

38. Andreux PA, Houtkooper RH, Auwerx J (2013) Pharmacological approaches to restore mitochondrial function. Nat Reu Drug Discou 12: 465- 483

39. Jin H, Kanthasamy A, Ghosh A, Anantharam V, Kalyanaraman B, Kanthasamy AC (2014) Mitochondria-targeted antioxidants for treatment of Parkinson's disease: preclinical and clinical outcomes. Biochim Biophys Acta 1842: 1282-1294

40. Yasuda T, Hayakawa H, Nihira T, Ren YR, Nakata Y, Nagai M, Hattori N, Miyake K, Takada M, Shimada T et al (2011) Parkin-mediated protection of dopaminergic neurons in a chronic MPTP-minipump mouse model of Parkinson disease. J Neuropathol Exp Neurol 70: 686-697

41. Hasegawa K, Yasuda T, Shiraishi C, Fujiwara K, Przedborski S, Mochizuki $\mathrm{H}$, Yoshikawa K (2016) Promotion of mitochondrial biogenesis by necdin protects neurons against mitochondrial insults. Nat Commun 7: 10943

42. Mudo G, Makela J, Di Liberto V, Tselykh TV, Olivieri M, Piepponen P, Eriksson O, Malkia A, Bonomo A, Kairisalo M et al (2012) Transgenic expression and activation of PGC-1alpha protect dopaminergic neurons in the MPTP mouse model of Parkinson's disease. Cell Mol Life Sci 69: 1153-1165

43. Angerer H (2015) Eukaryotic LYR proteins interact with mitochondrial protein complexes. Biology 4: 133-150

44. Hashimoto R, Nakazawa T, Tsurusaki Y, Yasuda Y, Nagayasu K, Matsumura K, Kawashima H, Yamamori H, Fujimoto M, Ohi K et al (2016) Whole-exome sequencing and neurite outgrowth analysis in autism spectrum disorder. J Hum Genet 61: 199-206

45. Frezza C, Cipolat S, Scorrano L (2007) Organelle isolation: functional mitochondria from mouse liver, muscle and cultured fibroblasts. Nat Protoc 2: $287-295$

46. Jha P, Wang X, Auwerx J (2016) Analysis of mitochondrial respiratory chain supercomplexes using blue native polyacrylamide gel electrophoresis (BN-PACE). Curr Protoc Mouse Biol 6: 1-14

47. Ago Y, Kawasaki T, Nashida T, Ota Y, Cong Y, Kitamoto M, Takahashi T, Takuma K, Matsuda T (2011) SEA0400, a specific $\mathrm{Na}^{+} / \mathrm{Ca}^{2+}$ exchange 
inhibitor, prevents dopaminergic neurotoxicity in an MPTP mouse model of Parkinson's disease. Neuropharmacology 61: 1441-1451

48. Kawasaki T, Ago Y, Kitao T, Nashida T, Takagi A, Takuma K, Matsuda T (2008) A neuroprotective agent, T-817MA (1-\{3-[2-(1-benzothiophen-5-yl) ethoxy]propyl\} azetidin-3-ol maleate), prevents 1-methyl-4-phenyl-1,2,3,6tetrahydropyridine-induced neurotoxicity in mice. Neuropharmacology 55: $654-660$

49. Seiriki K, Kasai A, Kuwaki T, Nakazawa T, Yamaguchi S, Hashimoto $H$ (2016) Critical involvement of the orbitofrontal cortex in hyperlocomotion induced by NMDA receptor blockade in mice. Biochem Biophys Res Commun 480: $558-563$

50. Lampl T, Crum JA, Davis TA, Milligan C, Del Gaizo Moore V (2015) Isolation and functional analysis of mitochondria from cultured cells and mouse tissue. J Vis Exp 97: e52076
51. Yamasaki A, Kasai A, Toi A, Kurita M, Kimoto S, Hayata-Takano A, Nakazawa T, Nagayasu K, Shintani N, Hashimoto R et al (2015) Identification of the role of bone morphogenetic protein (BMP) and transforming growth factor-beta (TGF-beta) signaling in the trajectory of serotonergic differentiation in a rapid assay in mouse embryonic stem cells in vitro. J Neurochem 132: $418-428$

52. Hashimoto H, Nogi H, Mori K, Ohishi H, Shigemoto R, Yamamoto K, Matsuda T, Mizuno N, Nagata S, Baba A (1996) Distribution of the mRNA for a pituitary adenylate cyclase-activating polypeptide receptor in the rat brain: an in situ hybridization study. J Comp Neurol 371: $567-577$

53. Mashiko D, Fujihara Y, Satouh Y, Miyata H, Isotani A, Ikawa M (2013) Generation of mutant mice by pronuclear injection of circular plasmid expressing Cas9 and single guided RNA. Sci Rep 3: 3355 